\title{
Muster der Landesgesetzgebung. Eine Analyse aller Landesgesetze zwischen 1990-2020
}

\author{
Christian Stecker · Jannis Kachel · Jana Paasch
}

Eingegangen: 14. Dezember 2020 / Überarbeitet: 7. März 2021 / Angenommen: 11. März 2021 / Online publiziert: 6. April 2021

(C) Der/die Autor(en) 2021

Zusammenfassung Die vorliegende Research Note stellt die erste systematische Dokumentation der Gesetzgebung in den deutschen Landtagen vor. Der Datensatz umfasst insgesamt 16.610 dokumentierte Gesetzgebungsvorgänge zwischen den Jahren 1990 und 2020. Nach einer Beschreibung des Datensatzes werden einige Gesetzgebungsmuster in den deutschen Ländern exemplarisch dargestellt. Die Landesgesetzgebung erweist sich dabei als stark durch den neuen Dualismus zwischen Regierung und Opposition geprägt. Im Initiativverhalten lassen sich zudem die Anreize des thematischen Parteienwettbewerbs ablesen. Wenig Evidenz findet sich für die These, dass innerkoalitionäre Gegensätze die Dauer der Gesetzgebungsverfahren in die Länge ziehen. Der mit dieser Research Note veröffentlichte Datensatz steht der Forschung für die Untersuchung zahlreicher weiterer Fragestellungen zur Verfügung.

Schlüsselwörter Gesetzgebung · Bundesländer · Landtage · Föderalismus · Mehrebenensystem

C. Stecker · J. Kachel

TU Darmstadt, Darmstadt, Deutschland

C. Stecker $(\square)$

Mannheimer Zentrum für Europäische Sozialforschung (MZES), Mannheim, Deutschland E-Mail: christian.stecker@tu-darmstadt.de

J. Paasch

Universität Potsdam, Potsdam, Deutschland

E-Mail: jana.paasch@uni-potsdam.de 


\title{
Patterns of Law-Making in the German States. An Analysis of All Bills Between 1990 and 2020
}

\begin{abstract}
This research note presents the first systematic documentation of the legislative process in the German state parliaments. The data set comprises 16,610 bills between 1990 and 2020. After a description of the data, we provide illustrative insights into the patterns of law-making. It is shown that these patterns are dominated by the new dualism between government and opposition. Furthermore, the incentives of issue competition are clearly present in the legislative initiatives. There is no evidence, however, for the expectation that intracoalitional policy distance prolongs the duration of legislative procedures. The published data provides scholars with the opportunity to investigate various additional research questions.
\end{abstract}

Keywords Legislative process $\cdot$ Regional states $\cdot$ Multilevel system $\cdot$ Federalism $\cdot$ Germany

\section{Einleitung}

Nach der Wahl der Repräsentanten bildet die Gesetzgebung die zweite Stufe des demokratischen Prozesses (Kielmansegg 2013, S. 42-49; McGann 2006), in dem die Vielfalt gesellschaftlicher Interessen in kollektiv verbindliche Entscheidungen gebündelt wird. An Gesetzgebungsverfahren lassen sich gleichsam wichtige Merkmale demokratischer Systeme und der darin agierenden Akteure ablesen. Es zeigt sich beispielsweise, welche Themen die politischen Repräsentantinnen und Repräsentanten aufgreifen (Jones und Baumgartner 2005), welche Konflikte zwischen ihnen bestehen (Kriesi et al. 2008; Lipset und Rokkan 1967) und wie inklusiv bzw. konsensual (Ganghof et al. 2019; Lijphart 1999; Müller und Jenny 2004, S. 311) darüber entschieden wird. Zudem enthält auch die Struktur und Dauer von Gesetzgebungsverfahren wichtige Informationen über die Performanz demokratischer Entscheidungssysteme (Reutter 2007; Schüttemeyer 2003; Sebaldt 2009, S. 87). Kurz: Die Analyse von Gesetzgebungsprozessen bietet wichtige Einblicke in die Interaktion politischer Akteure, in die Themen- und Konfliktstruktur sowie in die Performanz demokratischer Systeme (Roller 2004).

Diese Aspekte wurden in zahlreichen Untersuchungen zur Bundesgesetzgebung bereits aufgehellt (z. B. König und Bräuninger 2005; Manow und Burkhart 2007; Reutter 2007). Über die Landesgesetzgebung liegen neben Übersichtsdarstellungen (z.B. Leunig 2012; Mielke und Reutter 2012b) einsichtsvolle Fallstudien zu ausgewählten Politikbereichen vor (z.B. Blumenthal 2009; Henkes und Kneip 2010; Leber 2014; Reus und Zohlnhöfer 2015). Eine Analyse der Muster der Landesgesetzgebung auf Basis vollständiger Daten stand aber bisher aus. Mit einem von der Deutschen Forschungsgemeinschaft (DFG) geförderten Projekt haben wir begonnen, diese Lücke zu füllen. Darin wurden Daten zu Inhalt, Verlauf und Entscheidungen in der Landesgesetzgebung zwischen 1990-2019 erhoben. Mit dieser Research Note möchten wir die Daten veröffentlichen und interessierten Forscherinnen und Forschern zur Verfügung stellen. Dabei präsentieren wir einige Kernbefunde, die im 
Lichte verschiedener Forschungsperspektiven besonders interessant erscheinen. Unser Augenmerk liegt dabei auf:

- den vom neuen Dualismus geprägten Initiativtätigkeiten und Annahmequoten von Gesetzentwürfen;

- den Themen der Gesetzentwürfe, bei denen unterschiedliche Schwerpunkte durch unterschiedliche Parteien gesetzt werden;

- Struktur und Dauer des Gesetzgebungsverfahrens, das wiederum durch Faktoren wie die inhaltliche Kohärenz von Koalitionsregierungen beeinflusst ist.

Unser Artikel und der in diesem Rahmen veröffentlichte Datensatz leisten verschiedene Beiträge. Zunächst füllen wir eine Dokumentationslücke zu den deutschen Landtagen. Den vollständigen Individualdaten zu Verlauf und Ergebnis der Gesetzgebung auf Bundesebene (Burkhart und Manow 2006; König und Bräuninger 2005; Stecker 2015, 2016) stehen für die Länder meist nur Aggregatstatistiken gegenüber (Mielke und Reutter 2012a; Reutter 2013). Eine jüngere Ausnahme stellt die Monografie von Stefan Schukraft (2019) dar. Nachdem wir uns ausführlich durch die bunte föderale Vielfalt der Informationssysteme einzelner Landtage gearbeitet haben, können wir nun einen Individualdatensatz der Landesgesetzgebung vorlegen. Wir hoffen, dass er sich für die Scientific Community für zahlreiche Fragestellungen als fruchtbar erweist. Zunächst ist die Landesgesetzgebung allein gegenstandsbezogen für alle von Interesse, die sich mit Länderpolitik beschäftigen (z. B. Hildebrandt und Wolf 2008; Sack und Töller 2018; Wenzelburger et al. 2020). Als ,,most-similar systems“ bieten die Länder zudem gute Bedingungen für die Analyse von Institutioneneffekten und Handlungsmustern. Methodisch hilfreich ist vor allem, dass die Länderparteiensysteme zwar Eigenarten aufweisen (Detterbeck und Renzsch 2008), aber weitgehend von denselben Parteien bespielt werden. Anders als z. B. in Spanien (León 2014) unterscheidet sich auch die Kompetenzverteilung vis-à-vis der Bundesebene im Rahmen des symmetrischen Föderalismus nicht. Je nach Fragestellung ergibt sich somit eine fruchtbare experimentelle Varianz und es ist gleichzeitig eine große Kontrolle externer Störfaktoren erreichbar, die im internationalen Ländervergleich nicht möglich ist (Döring 1995; Jeffery und Schakel 2012; Snyder 2001). Im „Länderlabor“ (Rohrschneider 1994) konnten entsprechend bereits interessante Einsichten zu Koalitionsbildungsprozessen (Debus und Müller 2013; Jun 1994), Parteienwettbewerb (Bräuninger et al. 2020; Müller 2013), Fraktionsgeschlossenheit (Schukraft 2011; Stecker 2013) oder einzelnen politischen und administrativen Entscheidungen (Henkes und Kneip 2010; Hörisch 2018; Schneider et al. 2020) gewonnen werden. Inwieweit die resultierenden Einblicke über die Bundesländer hinaus als systematische Effekte verallgemeinerbar sind, ist freilich eine Frage, die es im Einzelfall sorgfältig zu prüfen gilt.

Neben dem Füllen der Dokumentationslücke liegt der substanzielle Beitrag unseres Artikels darin, dass wir hier einige interessante Fragestellungen bereits selbst beleuchten. So werden wir aufhellen, wie sich die für die parlamentarischen Systeme der Länder typischen Interaktionen der Parteien in den Gesetzgebungsmustern niederschlagen. Konkret untersuchen wir die vielfältigen Implikationen von Coalition Governance (Kropp 2001) und die Interaktion zwischen Regierung und Opposition (Kalke und Raschke 2004) mit Blick auf die Einbringung und Verabschiedung von 
Gesetzen und den Wettbewerb um die Wichtigkeit von Themen. Die resultierenden Befunde fügen ein weiteres Puzzlestück zu unserem Verständnis der Demokratiemuster der Länder (Freitag und Vatter 2008; Wehling 2002) und der Leistungen des deutschen Föderalismus und seiner Reformbedarfe hinzu (Benz 2009; Blancke 2004; Decker 2011; Kropp 2010; Wachendorfer-Schmidt 2005).

Dass die unitarische Entwicklung des deutschen Föderalismus zu einer deutlichen Aushöhlung bzw. Verwaltungslastigkeit der Länderkompetenzen geführt haben mag (Abromeit 1992; Hesse 1962), welche auch durch die jüngste Föderalismusreform nicht gravierend verändert wurde (Behnke und Kropp 2016; Stecker 2016), schmälert dabei die Aussagekraft unserer Ergebnisse nicht. Auf der einen Seite kann damit der Umfang der Aushöhlung selbst genauer beleuchtet werden - auch wenn wir dies hier selbst nicht genauer tun. Auf der anderen Seite (zusammenfassend Decker 2011, S. 284-285) beinhaltet auch die Ausführung von Bundesgesetzen politische Elemente (Leunig und Träger 2012, S. 20) bzw. gelten die verbliebenen Kompetenzen (insbesondere die Schulpolitik) als ,hochpolitisch“ und werden von den Länderregierungen auch genutzt, um eigene materielle Politikkonzepte zu verwirklichen (z. B. Reus und Vogel 2018; Sack 2010; Töller und Roßegger 2018).

Nachdem wir den Datensatz zur Landesgesetzgebung genauer vorgestellt haben, werden einige dieser Aspekte genauer unter die Lupe genommen. Dazu diskutieren wir zunächst die jeweilige Literatur und präsentieren direkt im Anschluss die interessierenden Muster in der Landesgesetzgebung. Wir testen hier also im engeren Sinne keine Hypothesen. Vielmehr wenden wir gut etabliertes Wissen (z. B. über die Wirkungen des neuen Dualismus) auf den Fall der Landesgesetzgebung an (vgl. zur Anwendung etablierter theoretischer Modelle Ganghof 2017). ${ }^{1}$

\section{Beschreibung des Datensatzes}

Ausgangspunkt unserer Datensammlung waren die (rohen) Daten zur Landesgesetzgebung, wie sie inzwischen von den Landtagen über die eigene Parlamentsdokumentation zur Verfügung gestellt werden. Dort können über Suchmasken die Gesetzgebungsvorgänge für einzelne Wahlperioden ausgegeben werden. Aus den resultierenden HTML- oder PDF-Dokumenten können die semistrukturierten Informationen computergestützt geerntet und in einen strukturierten Datensatz überführt werden. Eine (weitere) Blüte des deutschen Föderalismus stellte hier jedoch eine sehr große Herausforderung dar: So wie die Bundesländer ihrer Eigenstaatlichkeit durch 16 distinkte Elterngeldformulare oder Corona-Verordnungen (Behnke 2020) Ausdruck verleihen, so unterhalten sie jeweils auch eigenständige Landtagsinformationssysteme, die die uns interessierenden Daten in unterschiedlicher Tiefe und in unterschiedlicher Struktur bereithalten. Der Sinn dieser föderalen Vielfalt erschließt sich nicht unmittelbar, in jedem Fall ist dies einer effizienten komparativen Analyse der Ländergesetzgebung hinderlich. Für jeden der 16 Landtage mussten maßgeschneiderte Erhebungsverfahren entworfen und implementiert werden, wobei nicht

\footnotetext{
1 Wir lösen uns daher auch von der konventionellen Struktur aus einem Teil mit Theorie- bzw. Hypothesen und der empirischen Anwendung.
} 
selten auch Varianz über Wahlperioden hinweg weitere Anpassungen erforderte. Ein wenig Trost bei dieser mühsamen Tätigkeit findet man allein im noch größeren Leid derjenigen, die sich um die maschinenlesbare Transformation von Plenarprotokollen auf Länderebene verdient machen (Blätte 2017).

Konkret wurden die Gesetzgebungsdaten über skriptbasierte Browsersteuerung (RSelenium) ausgegeben und anschließend mit Web-Scraping- (rvest, xml2) und Pattern-Matching-Techniken (stringi, tidyverse) geerntet und in strukturierte rechteckige Datensätze überführt. Die gesamte Erhebungsinfrastruktur wurde in der Programmiersprache $\mathrm{R}$ aufgesetzt. Neben den Verfassern des vorliegenden Artikels haben dazu insbesondere Cenchen Liu, Kheoni Son und Marius Sältzer wichtige Beiträge geleistet.

Um eine vergleichende Analyse zu ermöglichen, mussten die Daten umfangreich harmonisiert und vereinheitlicht werden. Beispielsweise wurden die unterschiedlichen Schreibweisen von Verfahrensständen (z.B. „Ablehnung“, ,abgelehnt“), von Fraktionen (z. B. „FDP“, ,F.D.P.“), Verfassungsinstitutionen (z. B. „LReg“, „Landesregierung“) und Ausschüssen in eine einheitliche Terminologie überführt. Mithilfe flankierender Datensätze zu Wahlergebnissen und Regierungszusammensetzungen (Bräuninger et al. 2020) wurde zudem bestimmt, welche Fraktionen in welchem Zeitraum der Regierung und welche der Opposition angehörten. Mit umfangreichen Checks, z. B. einem Vergleich mit offiziellen Aggregatstatistiken zur Gesetzgebung, konnte eine hinreichende Genauigkeit der Daten verifiziert werden. Es ist aber praktisch ausgeschlossen, dass der Datensatz fehlerfrei ist. Daher freuen sich die Ver-

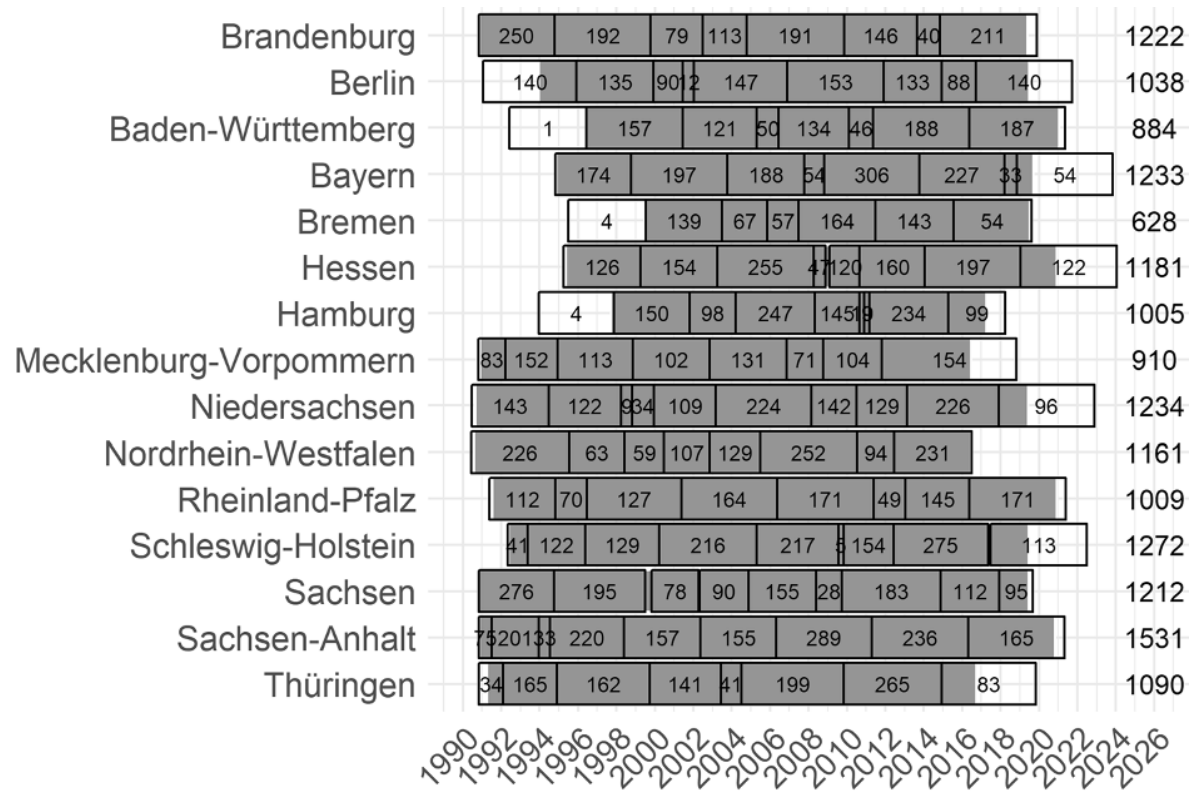

$n=16610$ Gesetze

Abb. 1 Anzahl der Gesetzgebungsvorgänge nach Bundesländern 
fasser nicht nur über eine breite Nutzung der Daten, sondern auch über sich dabei ergebende Hinweise auf Fehler. Die im Artikel verwendete Version des Datensatzes ist im harvard dataverse ${ }^{2}$ abrufbar. Aktualisierungen werden auf einer Homepage ${ }^{3}$ veröffentlicht.

Die Daten sind zum Stand 16.11.2020 zur jeweils letzten abgeschlossenen Legislaturperiode eines Landtages vollständig. Abb. 1 fasst wichtige Aspekte zusammen.

Insgesamt umfasst der Datensatz 16.610 Gesetzgebungsvorgänge zwischen den Jahren 1990 und 2020. Aufsummiert ergeben sich Informationen für insgesamt 117 Länderkabinette. Dabei sind einzelne Länder mit unterschiedlicher Dauer vertreten. Beispielsweise könnten für Nordrhein-Westfalen Daten bereits ab 1985 präsentiert werden. ${ }^{4}$ In Bayern liegen Gesetzgebungsdaten in einer maschinenlesbaren Form dagegen erst ab 1994 vor.

Für jeden Gesetzgebungsvorgang wurden umfangreiche Informationen erhoben, insbesondere zu den Initiatoren, zum Datum verschiedener Verfahrensschritte (z. B. Einbringung, Lesungen, Abstimmungen), zum Politikfeld bzw. den beteiligten Ausschüssen und zum Ergebnis des Gesetzgebungsprozesses.

\section{Gesetzgebungsmuster in den deutschen Ländern}

\subsection{Landesgesetzgebung im neuen Dualismus parlamentarischer Regierungssysteme}

Die deutschen Länder sind parlamentarische Regierungssysteme meist mit Koalitionsregierungen (Leunig 2012). In parlamentarischen Regierungssystemen ist der Dualismus zwischen Regierung und Opposition der zentrale Prägefaktor zahlreicher politischer Prozesse (bereits Bagehot 2001 [1867], S. 11, 14; Dewan und Spirling 2011). Dieser Dualismus und die dadurch strukturierte Interaktion von Parteien (bzw. Fraktionen) innerhalb der Regierung und zwischen Regierung und Opposition wirkt auch entscheidend auf die Muster der Gesetzgebung - also auf Urheberschaft, Anzahl, Inhalt, Beginn, Verlauf und Abschluss einzelner Gesetzgebungsverfahren sowie die sich dabei bildenden Gesetzgebungskoalitionen (König und Bräuninger 2005). Um die Gesetzgebungsmuster in einem parlamentarischen System zu analysieren, müssen daher insbesondere zwei Akteurskonstellationen (Scharpf 2006, S. 87) bzw. Interaktionsebenen in den Blick genommen werden: die Interaktion zwischen Parteien innerhalb der Regierung und die Interaktion zwischen Regierungsund Oppositionsparteien.

\footnotetext{
2 https://dataverse.harvard.edu/dataset.xhtml?persistentId=doi\%3A10.7910\%2FDVN\%2FOWZ53K\& version=DRAFT.

3 www.chrstecker.de.

4 Da erst ab 1990 flankierende Daten, z. B. zu Parteipositionen vorliegen, wurde der Datensatz mit Beginn vom 01.01.1990 konsolidiert.
} 


\subsubsection{Interaktion zwischen Regierung und Opposition}

Der Dualismus in parlamentarischen Systemen impliziert eine klare Machtverteilung zwischen Regierung und Opposition ${ }^{5}$ und prägt auch entscheidend die Interaktion zwischen Regierungsmehrheit und Opposition. Auf beiden Seiten des neuen Dualismus wirken starke Anreize, sich voneinander abzugrenzen. Die Regierung sollte kraft eigener Mehrheit regieren, also ihre Gesetzgebungsagenda erfolgreich durch das Parlament führen und sich damit als handlungsfähig erweisen. Das primäre Ziel der Opposition ist es, sich als klare Alternative zur Regierung zu präsentieren (Brader et al. 2013, S. 1490). Diese rollenspezifischen Verhaltensmuster lassen sich an verschiedenen Stadien der Gesetzgebung ablesen. So ist für die Bundesgesetzgebung bekannt, dass Entwürfe meist entweder von der Regierung oder der Opposition bzw. einer Oppositionspartei eingebracht werden (Ismayr 2008a; Rudzio 2015, S. 244). Oppositionsparteien nutzen (letztlich oft aussichtslose) Gesetzesinitiativen, Anträge und Änderungsanträge, um ihren alternativen Positionen Gehör zu verschaffen (Brunner 2013).

Abb. 2 veranschaulicht diese klare Rollenverteilung für die Landtage. Die Boxplots präsentieren zum einen die relativen Anteile bei der Einbringung aller Gesetzentwürfe (hellgrau), zum anderen die Anteile an den erfolgreichen Entwürfen

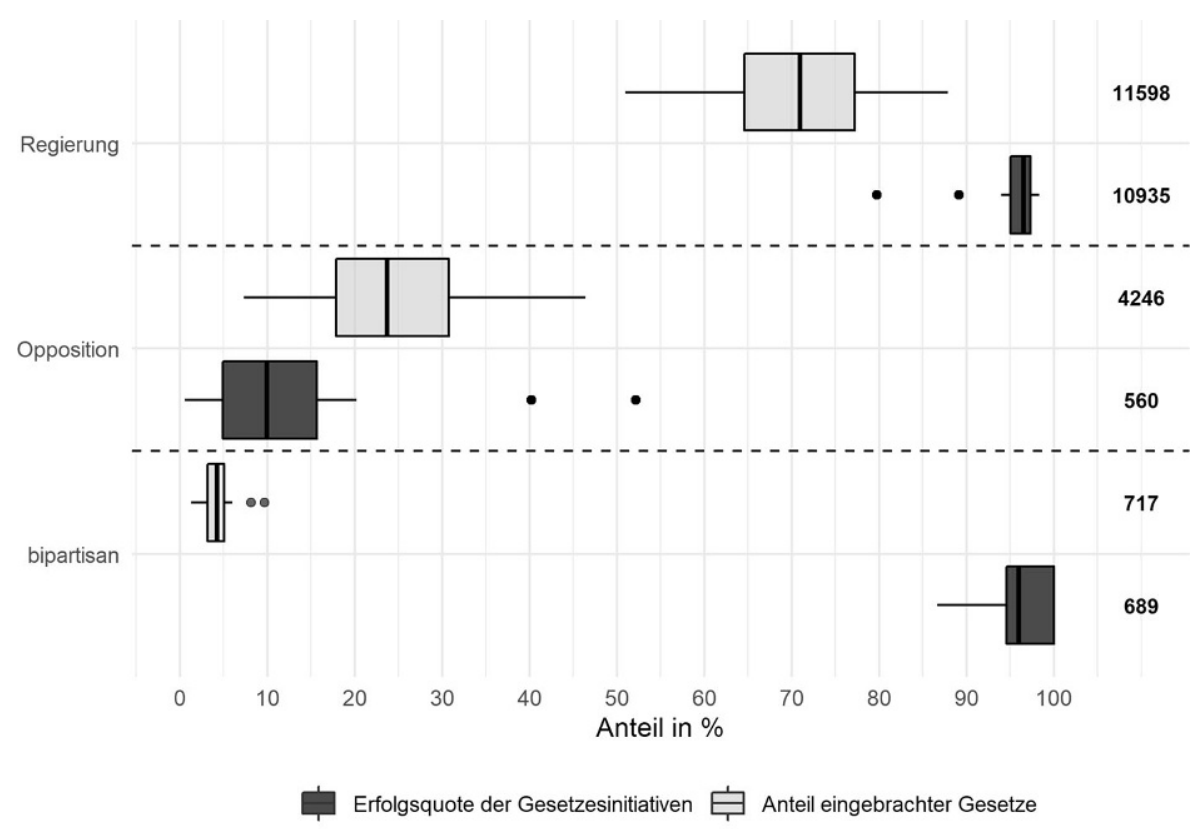

$n=16561$ von 16610 Gesetzen mit kategorisiertem Urheber

Abb. 2 Initiativtätigkeit und Erfolg von Regierung und Opposition

\footnotetext{
${ }^{5}$ Unserer Argumentation liegt hier das für die Bundesrepublik typische Vorhandensein von Minimalgewinnkoalitionen zugrunde.
} 
(dunkelgrau). Genauer gesagt, zeigen sie die Verteilung der für jedes Bundesland errechneten Anteile. Die Dominanz der Regierungsinitiative ist offensichtlich. So gehen im Median $71 \%$ der Entwürfe auf die Landesregierung bzw. auf die sie tragenden Fraktionen zurück. Von den Oppositionsfraktionen werden $24 \%$ der Entwürfe auf den parlamentarischen Weg gebracht. Überparteiliche Entwürfe ${ }^{6}$ aus der Mitte des Parlaments sind für circa $5 \%$ der insgesamt 16.224 Gesetze verantwortlich. Vollends sichtbar wird die Prägekraft des neuen Dualismus, wenn man die Annahmequoten der Gesetzentwürfe betrachtet. Tatsächlich und wenig überraschend besitzt die Regierung auch auf Landesebene ein Monopol in der Gesetzgebung: Etwa $92 \%$ der insgesamt 11.814 verabschiedeten Gesetzentwürfe gehen auf Regierungsinitiativen zurück. Die geringe Annahmequote der Oppositionsgesetze von durchschnittlich circa $14 \%$ (bezogen auf alle Oppositionsinitiativen) weist darauf hin, dass es hier vor allem darum geht, Alternativen zu präsentieren, diese aber kaum eine Chance auf parlamentarische Mehrheiten haben. Dagegen legt der große Erfolg interfraktioneller Entwürfe nahe, dass die gemeinsame Einbringung über Regierung und Opposition hinweg bereits einen breiten inhaltlichen Konsens vorwegnimmt.

Abb. 2 zeigt jedoch auch eine nennenswerte Varianz in den Anteilen der Initiativen über die einzelnen Bundesländer hinweg, welche möglicherweise substanziell interessanten Erklärungen zugänglich sind. Mögliche Teilerklärungen könnten in der Interaktion innerhalb der Regierung und zwischen den Regierungs- und Oppositionsparteien liegen, auf die wir im Folgenden eingehen.

\subsubsection{Interaktion innerhalb der Regierung}

Wie unsere Daten zeigen, besitzen die Landesregierungen - wie es typisch für parlamentarische Systeme ist - durch die (häufige) Kontrolle einer disziplinierten Parlamentsmehrheit und der Ressourcen der Ministerialbürokratie ein Quasimonopol in der Gesetzgebung (Ismayr 2008b; Tsebelis 2002, S. 82). Aufgrund ihrer Dominanz ist die Regierung selbst wiederum ein wichtiger Faktor, um Gesetzgebungsmuster zu erklären (Guinaudeau und Guinaudeau 2020). Wichtige Unterschiede verlaufen hier zwischen Mehrheits- und Minderheits- bzw. zwischen Einparteien- und Koalitionsregierungen. Mit Blick auf die in den Landtagen häufigen Koalitionsregierungen sind wiederum die politischen Gegensätze zwischen den Koalitionspartnern relevant. Diesen Gegensätzen wurde in verschiedenen Studien zugeschrieben, ganz unterschiedliche Aspekte von Gesetzgebungsprojekten zu beeinflussen.

So wurde anhand verschiedener parlamentarischer Systeme gezeigt, dass die Gesetzgebungstätigkeit der Regierung bei wachsenden internen Gegensätzen sinkt (Bräuninger und Debus 2009, S. 811; Däubler 2008, S. 697; Tsebelis 1999). Anhand einer Stichprobe individueller Gesetze aus vier Ländern beobachtet Martin (2004), dass Vorhaben früher in der Legislaturperiode auf den Weg gebracht werden, wenn sich die Regierungsparteien über deren Wichtigkeit und Inhalt einig sind. Strittige Vorlagen schieben sie dagegen auf die längere Bank. Auch die Dauer des Verfahrens bzw. bestimmter Verfahrensschritte (Martin und Vanberg 2004) sowie Häufigkeit und

\footnotetext{
6 Alle Entwürfe, die mindestens von einer Regierungsfraktion und einer Oppositionsfraktion eingebracht werden, kategorisieren wir als überparteilich.
} 
Umfang von Änderungen in der parlamentarischen Befassung (Martin und Vanberg 2005) sind mit der politischen Distanz innerhalb der Regierung korreliert. Je mehr Konflikt in der Regierung, so eine Kerneinsicht der viel rezipierten Studien von Martin und Vanberg, desto länger dauert die Befassung und desto häufiger kommt es zu inhaltlichen Änderungen in der Ausschussphase. Ursächlich ist, dass das Regieren in Koalitionen ein Spiel mit gemischten Motiven ist (Boston und Bullock 2012). Auf der einen Seite möchten die Koalitionsparteien effizient gemeinsam regieren, um von der kollektiven Reputation zu profitieren und um handlungsfähig zu sein. Auf der anderen Seite stehen die Koalitionsparteien im Wettbewerb um Wählerstimmen gegeneinander und müssen sich dazu auch vom Koalitionspartner abgrenzen, um für die Wählerinnen und Wähler sichtbar zu bleiben. Die inhaltliche Heterogenität beeinflusst in diesem Spiel sowohl die Einigungsfähigkeit der Regierungsparteien als auch wie intensiv sich Koalitionspartner gegenseitig auf die Einhaltung von Koalitionskompromissen in ihren jeweiligen ministeriellen Portfolios kontrollieren müssen (Strøm et al. 2008). Dabei ist das Ausmaß der inhaltlichen Heterogenität zum Teil mit dem Regierungs- bzw. Koalitionsformat verbunden (siehe auch Kalke und Raschke 2004). Martin und Vanberg nehmen explizit an, dass die intrakoalitionäre Kontrolle in den Parlamentsausschüssen stattfindet und sich daher an einer längeren Ausschussphase (und umfangreicheren Änderungen) ablesen ließe. Freilich widerspricht diese Annahme dem gut gesicherten qualitativen Wissen über die Ausschüsse und es ist eher zu vermuten, dass die Lösung von Konflikten den vielfältigen informellen Koordinationsmechanismen von Koalitionen überlassen bleibt (Miller 2011; Saalfeld 2000). ${ }^{7}$ Auch eine intensivere innerkoalitionäre Koordination könnte sich jedoch in verlängerten Gesetzgebungsverfahren niederschlagen - sofern sie nicht vollständig dem Kabinettsbeschluss zur Einbringung einer Initiative vorangeht. In jedem Fall erscheint es lohnenswert, die Daten im Lichte dieser Intuition zu befragen.

Dazu haben wir ausgewertet, inwieweit die inhaltliche Heterogenität der Regierung Anzahl und Dauer ihrer Gesetzgebungsinitiativen beeinflusst. Der Datensatz wird dazu auf die Regierungsgesetze reduziert und die durchschnittliche Dauer des Gesetzgebungsprozesses ermittelt. Um die inhaltliche Heterogenität zu berechnen, greifen wir auf Daten zu den wirtschafts- und gesellschaftspolitischen Positionen der Landesparteien zurück, welche die beiden zentralen Konfliktlinien in der Bundesrepublik repräsentieren. Diese Daten wurden von Bräuninger et al. (2020) mithilfe der Wordscores-Methode (Laver et al. 2003) aus einem umfassenden Korpus aller Wahlprogramme auf Bundes- und Länderebene gewonnen. Die inhaltliche Heterogenität einer Landesregierung haben wir als die durchschnittliche euklidische Distanz im

\footnotetext{
7 Grundsätzlich kann an den - zumindest in der internationalen Literatur - sehr erfolgreichen Studien kritisiert werden, dass die quantitativen Ergebnisse unzureichend mit dem umfassenden qualitativ fundierten Wissen über Gesetzgebungsprozesse verknüpft sind. So nehmen Martin und Vanberg auch für den Deutschen Bundestag an, dass sich die Koalitionsparteien in Parlamentsausschüssen unter den Augen der Opposition gegenseitig auf die Einhaltung von Kompromissen kontrollieren und dort Konflikte austragen und Änderungen durchsetzen. Dagegen zeigen qualitative Studien zumindest für Deutschland auf Bundesund Länderebene, dass Ausschüsse zuvorderst ein Ort der Konfrontation zwischen Regierung und Opposition sind (von Oertzen 2006; Schüttemeyer 1998) und regierungsinterne Auseinandersetzungen jenseits formaler parlamentarischer Institutionen ausgetragen werden (Miller 2011; Rudzio 2005).
} 


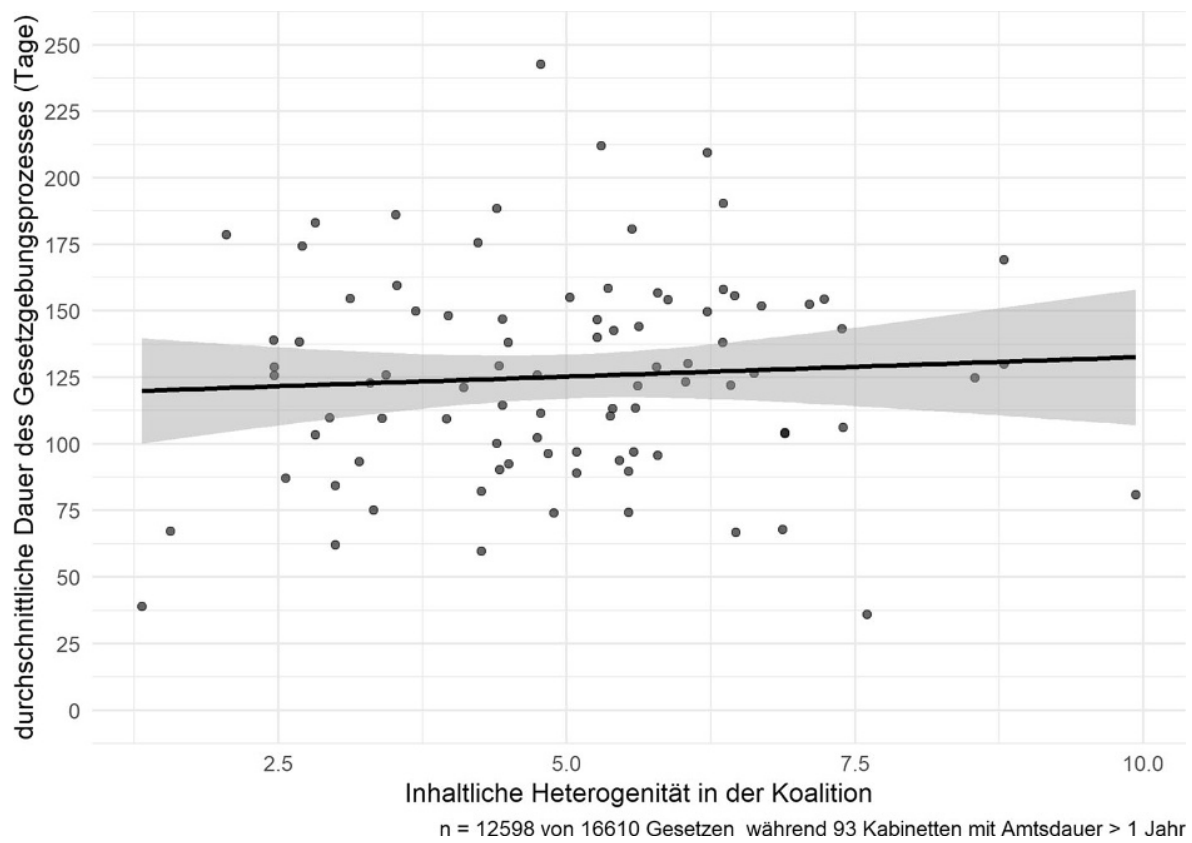

Abb. 3 Beeinflusst innerkoalitionäre Heterogenität die Dauer des Gesetzgebungsverfahrens von Regierungsinitiativen?

Quadrat (EDQ) der jeweiligen Regierungsparteien untereinander in einem zweidimensionalen Policy-Raum definiert. Je kleiner die durchschnittliche EDQ zwischen den Regierungsparteien, desto inhaltlich homogener ist sie.

Abb. 3 beleuchtet eine wichtige beobachtbare Implikation der Coalition-Governance-Hypothesen. Auf der X-Achse ist die inhaltliche Distanz innerhalb der Koalitionen abgetragen, die $y$-Achse wird durch die Dauer des Gesetzgebungsprozesses in Tagen aufgespannt. Wie Martin und Vanberg erwarten würden, lässt sich ein negativer Einfluss regierungsinternen Konflikts auf die Verfahrensdauer identifizieren. Kabinette, die - zumindest nach unserer Messung - wenig inhaltliche Stolpersteine aus dem Weg räumen müssen, sind im Schnitt zügiger als Kabinette, bei denen inhaltlicher Konflikt zum Koalitionsalltag gehört. Ebenso verhält es sich übrigens beim Einbringen von Regierungsinitiativen: Ihr Anteil sinkt, wenn die Distanz zwischen den Partnern steigt. Allerdings sind die Zusammenhänge schwach und sie verschwinden, wenn wir die Anzahl der Regierungsparteien als alternative Konfliktmessung zugrunde legen. Als Erklärung erscheint uns der bekannte Umstand plausibel, dass Kabinette Gesetzgebungsverfahren erst auf den Weg bringen, wenn sie sich über den Inhalt grundsätzlich einig geworden sind. 


\subsubsection{Interaktion zwischen Regierung und Oppositionsfraktionen im thematischen Themenwettbewerb}

In Mehrparteiensystemen kann der Gegensatz zwischen Regierung und Opposition natürlich nicht alle Nuancen in den Gesetzgebungsmustern aufhellen. Vor allem mit Blick auf die Oppositionsparteien ist die Aggregation in „die Opposition“ unzureichend, da hier unterschiedliche Fraktionen ohne das gemeinsame Band der Regierungsbeteiligung eigene Ziele verfolgen. Individuellen Parteistrategien und der dadurch konstituierte Parteienwettbewerb wirken auf unterschiedliche Aspekte. Dabei rückt neben dem Wettbewerb um Positionen zu einzelnen Themen der Wettbewerb um die Aufmerksamkeit für bestimmte Themen selbst in den Vordergrund. Die hier wirkenden Dynamiken wurden in der Literatur zu Issue Competition umfangreich analysiert (Jones und Baumgartner 2005; Petrocik 1996; Robertson 1976). Die Grundidee ist, dass Parteien interessengeleitet bestimmte Themen in den Vordergrund spielen, bei denen Nutzen und Schaden von Wähleraufmerksamkeit ungleich über die Parteien verteilt sind. Aufmerksamkeit für eine hohe Arbeitslosenquote dürfte beispielsweise eher den dafür mitverantwortlich gemachten Regierungsparteien schaden.

Zudem können Parteien eine Issue Ownership (Bélanger 2003; Walgrave et al. 2009) erwerben, das heißt, dass sie von den Wählern mit spezifischen Themen assoziiert werden (Busemeyer et al. 2013; Spoon et al. 2014). Eine Partei kann dabei sowohl von der assoziativen Verknüpfung eines politischen Themas durch die Wähler als auch von einer gesteigerten Wahrnehmung der Lösungskompetenz im Vergleich zu anderen Parteien profitieren (Budge 2015, S. 774; Dolezal et al. 2014, S. 60). Den an diesen Themen interessierten Wählern gelten sie als glaubwürdige und kompetente Vertreter der eigenen Interessen, was sich an der Wahlurne bezahlt machen dürfte. Der enge Nexus zwischen den Grünen und dem Umweltthema ist ein paradigmatisches Beispiel.

Diese grundlegende Handlungslogik wirkt sich auf verschiedene Aktivitäten von Parteien und ihren Fraktionen aus. Issue Competition schlägt sich in Wahlprogrammen (Green-Pedersen 2007) nieder und lässt sich an kleinen Anfragen (Proksch und Slapin 2011) oder Regierungserklärungen (Breunig und Schnatterer 2020) ablesen. Freilich können Parteien auch in der Gesetzgebung ihren Schwerpunkt auf die „richtigen“ Themen legen, welche ihr strategische Vorteile im Wahlwettbewerb verschaffen. Gesetzentwürfe eignen sich besonders dafür, da sie Zeit im Plenum und in den Ausschüssen beanspruchen und damit auch stärker ins Licht der Öffentlichkeit rücken. Im Sinne eines Themenwettstreits zwischen den Parteien ist also anzunehmen, dass die Parteien ihre Gesetzgebungstätigkeit in den Landtagen entlang ihrer Kernthemen ausrichten werden. Demnach werden Parteien Gesetzentwürfe einbringen, die den Wettbewerb um politische Themen reflektieren und ihre ,richtigen“ Themen in den Vordergrund rücken lassen. Im Umkehrschluss sollten die Akteure auch bemüht sein, unvorteilhafte Themen nicht auf die politische Agenda zu setzen (Busemeyer et al. 2013, S. 528; Green-Pedersen und Mortensen 2014, S. 760).

Welche Themen von einzelnen Parteien besetzt werden, variiert u.a. entlang ihrer Verortung auf der Rechts-Links-Dimension des Parteienwettbewerbs. Während die Parteien des linken Spektrums vornehmlich mit politischen Themen wie Wohl- 


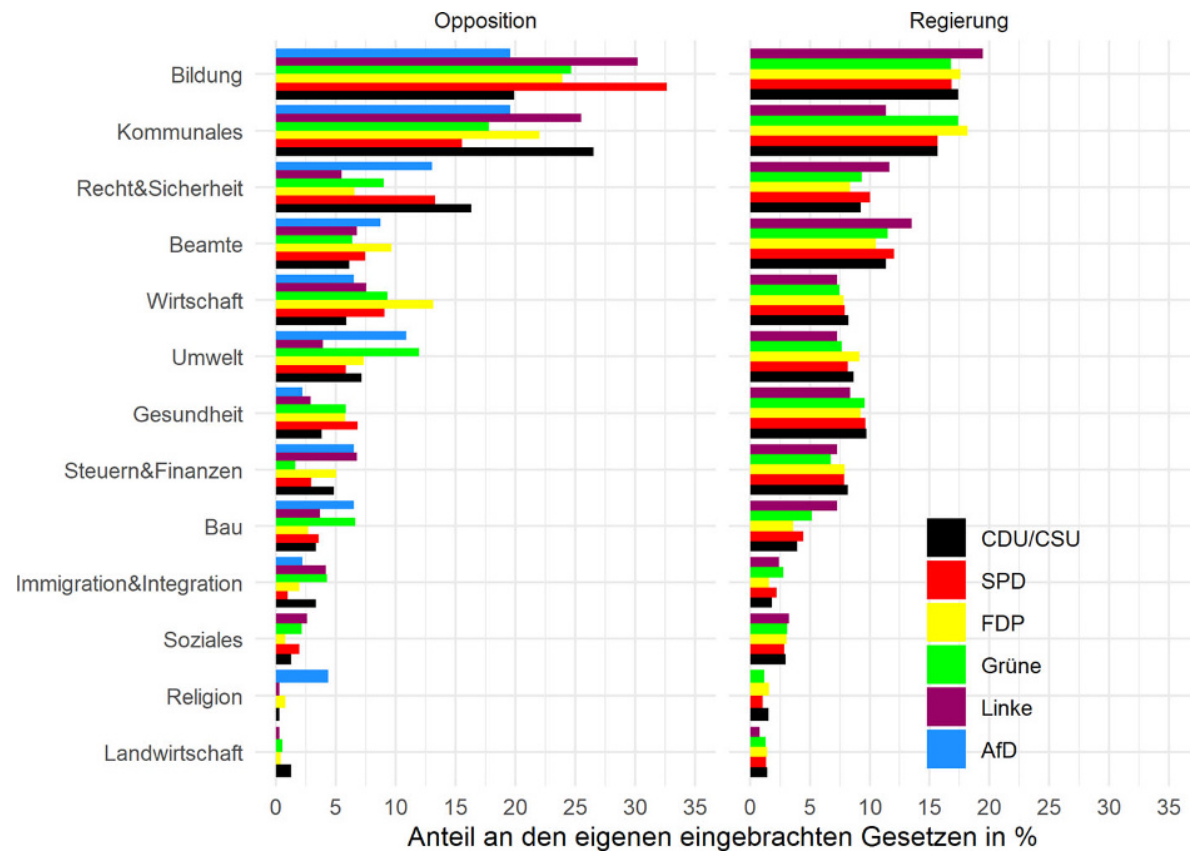

Abb. 4 Thematischer Parteienwettbewerb und Gesetzesinitiativen

fahrt, Arbeitslosigkeit, Arbeitsmarkt und Bildung verknüpft werden, assoziieren die Wähler die Parteien des rechten Spektrums eher mit den politischen Themen wie Wirtschaft, Sicherheit und Verteidigung. Darüber hinaus besetzen Parteien wie Die Grünen/Bündnis 90 die Umweltpolitik (Spoon et al. 2014; Switek 2017). Die Assoziation von Parteien mit bestimmten politischen Themen besagt jedoch wenig über die Wahrnehmung der Fähigkeiten einer Partei, Lösungen für die Politikgestaltung im jeweiligen Themenfeld anzubieten. Vielmehr verbinden die Wähler Parteien mit politischen Themen, ohne damit eine konkrete Lösungskompetenz zu unterstellen (Budge 2015, S. 765).

Abb. 4 beleuchte die Gesetzgebungsmuster aus der diskutierten Issue-Competition-Perspektive. Wir haben dazu 15.752 Gesetze manuell einer an den CAP-Kategorien orientierten Klassifikation zugeordnet. Für die Kodierentscheidungen wurden die Titel der Gesetze betrachtet und, sofern vorhanden, die Synopse des Vorhabens konsultiert. Häufig betreffen Gesetze zwei oder mehr Politikbereiche. Ausgewählt wurde der Bereich, der am stärksten von einem Gesetz berührt wurde. Diese Arbeit wurde von zwei Kodierern unabhängig voneinander bewältigt. Ihre Entscheidungen wiesen eine sehr hohe Übereinstimmung auf, die wenigen gegenläufigen Kodierungen wurden in einer gemeinsamen Diskussion harmonisiert.

Die Balken zeigen jeweils die Anteile der Politikbereiche unter den von einer Partei (mit)eingebrachten Gesetzen und geben damit Aufschluss, ob unterschiedli- 
che Parteien auch unterschiedliche Politikbereiche betonen. ${ }^{8}$ Die Darstellung erfolgt getrennt für die Regierungs- und Oppositionsphasen der jeweiligen Parteien bzw. Fraktionen. Dahinter steht die Intuition, dass Regierungs- und Oppositionsstatus unterschiedliche Freiheitsgrade gewähren, wenn mit Gesetzentwürfen thematischer Parteienwettbewerb betrieben werden soll. Regierungsfraktionen unterliegen klaren Sachzwängen bei der Initiativtätigkeit. So ist es für die Regierung u.a. nicht fakultativ, regelmäßig einen Haushalt einzubringen oder Gesetze des Bundes bzw. Richtlinien der Europäischen Union umzusetzen (Paasch und Stecker 2021). Zudem müssen im Normalfall einer Regierungskoalition die Gesetzentwürfe mit einem weiteren Partner abgestimmt werden, der oftmals andere Themenprioritäten verfolgen dürfte (Sagarzazu und Klüver 2017). Von einer allgemeinen gesetzgeberischen Bringschuld befreit, können Oppositionsfraktionen dagegen recht frei und individuell entscheiden, welche Themen sie mit eigenen Entwürfen bespielen möchten.

Diese Intuition wird von den Mustern bestätigt. Mit Blick auf die Regierungsseite zeigen sich nur marginale Differenzen zwischen den Parteien. Einer Regierungsfraktion erscheint die Gesetzgebung zur thematischen Schwerpunktsetzung weniger zugänglich und sie muss dazu z. B. auf Wahlprogramme oder Anfragen ausweichen (Breunig und Schnatterer 2020). In der Opposition setzen die jeweiligen Fraktionen aber klar unterschiedliche Akzente, die wiederum häufig mit typischen Erwartungen korrespondieren. So sind die Grünen im Bereich Umwelt besonders aktiv und gemeinsam mit der Linken auch bei Bürgerrechten/Gleichstellung. Die Union dominiert wiederum im Bereich Recht\&Sicherheit.

Interessant ist zudem, dass die Kompetenzverteilung zwischen Bund und Ländern in der Verteilung durchscheint. Gesetze zur Bildung, eine der wenigen eigenen Kernkompetenzen der Länder, werden besonders häufig von allen Parteien eingebracht. Die Häufigkeit der Kategorie „Kommunales“ und „Beamte“ dürfte ähnlich begründet sein.

\section{Zusammenfassung und Schluss}

Unser Beitrag präsentiert die erste systematische Dokumentation der Gesetzgebung in den deutschen Landtagen. Für insgesamt 16.610 Gesetzgebungsvorgänge zwischen den Jahren 1990 und 2020, während der Amtszeit von 117 Länderkabinetten, liegen damit Informationen zu den Initiatoren, zu den betroffenen Politikfeldern, zum Verlauf und zum Ergebnis vor. Neben der Präsentation des nun veröffentlichten Datensatzes haben wir schlaglichtartig sein Erkenntnispotenzial illustriert. Dazu wurden die Daten nach typischen Mustern befragt, die sich aus dem neuen Dualismus, der Logik von Koalitionsregierungen und dem thematischen Parteienwettbewerb ergeben. Es versteht sich von selbst, dass jeder dieser Aspekte eine eigene sorgfältige Studie verdient hat.

Beispielsweise widersprechen die recht schwachen Effekte der programmatischen Distanz zwischen den Koalitionspartnern auf die Dauer des Gesetzgebungsprozesses

\footnotetext{
${ }^{8}$ In der Zählung wurden die Entwürfe berücksichtigt, die eine Fraktion allein oder gemeinsam mit weiteren Fraktionen eingebracht hat.
} 
vielzitierten internationalen Studien zu Coalition Governance (Martin und Vanberg 2011). Freilich könnte unser Ergebnis damit zusammenhängen, dass die Klärung innerkoalitionären Dissenses im vorparlamentarischen Raum geschieht. Zum einen werden in Koalitionsverträgen zunehmend detailliertere Kompromisse verschriftlicht. Zum anderen sind Gesetze mit der „Kabinettsreife“ bereits so konfliktbereinigt, dass im parlamentarischen Prozess Verzögerungen unwahrscheinlich sind. Künftige Forschung könnte eruieren, inwiefern programmatische Distanz dann auf die parlamentarische Befassung wirkt, wenn detaillierte inhaltliche Vorklärung, z. B. in Koalitionsverträgen, nicht stattgefunden hat (Bowler et al. 2016).

Auch könnte weiter ausgeleuchtet werden, wie elektorale Anreize auf die Landesgesetzgebung wirken. Beispielsweise wäre es denkbar, dass die Muster der Issue Competition besonders im Vorfeld von Wahlen zutage treten. Schließlich folgen aus vielen Theorien der Policy-Forschung Implikationen, die sich an den Landesgesetzgebungsmustern ablesen lassen dürften. Mit dieser Research Note hoffen wir die Grundlage zu verbreitern, auf der die Länderparlamente als Teil des deutschen Mehrebenensystems künftig noch detaillierter untersucht werden können.

Danksagung Für wertvolle Unterstützung bei der computergestützten Erhebung der Daten danken wir: Cenchen Liu, Kheoni Son, Isabelle Kohl, Marius Michel, Georg Heilmann, Felix-Peer Caspari, Maximilian Haag, Marina König, Dylan Paltra und Marius Sältzer.

Funding Open Access funding enabled and organized by Projekt DEAL.

Open Access Dieser Artikel wird unter der Creative Commons Namensnennung 4.0 International Lizenz veröffentlicht, welche die Nutzung, Vervielfältigung, Bearbeitung, Verbreitung und Wiedergabe in jeglichem Medium und Format erlaubt, sofern Sie den/die ursprünglichen Autor(en) und die Quelle ordnungsgemäß nennen, einen Link zur Creative Commons Lizenz beifügen und angeben, ob Änderungen vorgenommen wurden.

Die in diesem Artikel enthaltenen Bilder und sonstiges Drittmaterial unterliegen ebenfalls der genannten Creative Commons Lizenz, sofern sich aus der Abbildungslegende nichts anderes ergibt. Sofern das betreffende Material nicht unter der genannten Creative Commons Lizenz steht und die betreffende Handlung nicht nach gesetzlichen Vorschriften erlaubt ist, ist für die oben aufgeführten Weiterverwendungen des Materials die Einwilligung des jeweiligen Rechteinhabers einzuholen.

Weitere Details zur Lizenz entnehmen Sie bitte der Lizenzinformation auf http://creativecommons.org/ licenses/by/4.0/deed.de.

\section{Literatur}

Abromeit, Heidrun. 1992. Der verkappte Einheitsstaat. Wiesbaden: Springer VS.

Bagehot, Walter. 2001. The English constitution (edited by Miles Taylor). New York: Oxford University Press.

Behnke, Nathalie, und Sabine Kropp. 2016. Arraying institutional layers in federalism reforms: lessons from the German case. Regional \& Federal Studies 26(5):585-602. https://doi.org/10.1080/ 13597566.2016.1236026.

Behnke, Nathalie. 2020. Föderalismus in der (Corona-)Krise? Föderale Funktionen, Kompetenzen und Entscheidungsprozesse. Aus Politik und Zeitgeschichte 70(35-37):9-15.

Bélanger, Éric. 2003. Issue ownership by Canadian political parties 1953-2001. Canadian Journal of Political Science 36(3):539-558. https://doi.org/10.1017/S0008423903778755.

Benz, Arthur. 2009. Politik in Mehrebenensystemen. Wiesbaden: VS.

Blancke, Susanne. 2004. Politikinnovationen im Schatten des Bundes. Policy-Innovationen und Diffusionen im Föderalismus und die Arbeitsmarktpolitik der Bundesländer. Wiesbaden: VS. 
Blätte, Andreas. 2017. Interaktion und Dialog mit großen Textdaten: Korpusanalyse mit polmineR. In Big Data. Große Möglichkeiten oder große Probleme?, Hrsg. Kai-Uwe Schnapp, Joachim Behnke, und Claudius Wagemann. Baden-Baden: Nomos.

von Blumenthal, Julia. 2009. Das Kopftuch in der Landesgesetzgebung Governance im Bundesstaat zwischen Unitarisierung und Föderalisierung. Baden-Baden: Nomos.

Boston, Jonathan, und David Bullock. 2012. Multi-party governance: managing the unity-distinctiveness dilemma in executive coalitions. Party Politics 18(3):349-368. https://doi.org/10.1177/ 1354068810382937.

Bowler, Shaun, Thomas Bräuninger, Marc Debus, und Indridi H. Indridason. 2016. Let's just agree to disagree: dispute resolution mechanisms in coalition agreements. The Journal of Politics 78(4):1264-1278. https://doi.org/10.1086/686805.

Brader, Ted, Joshua A. Tucker, und Dominik Duell. 2013. Which parties can lead opinion? Experimental evidence on partisan cue taking in multiparty democracies. Comparative Political Studies 46(11):1485-1517. https://doi.org/10.1177/0010414012453452.

Bräuninger, Thomas, und Marc Debus. 2009. Legislative agenda-setting in parliamentary democracies. European Journal of Political Research 48:804-839. https://doi.org/10.1111/j.1475-6765.2009.00850. $\mathrm{x}$.

Bräuninger, Thomas, Marc Debus, Jochen Müller, und Christian Stecker. 2020. Parteienwettbewerb in den deutschen Bundesländern. Wiesbaden: Springer VS.

Breunig, Christian, und Tinette Schnatterer. 2020. Die politische Agenda Deutschlands. Politische Vierteljahresschrift 61(1):131-149. https://doi.org/10.1007/s11615-020-00226-6.

Brunner, Martin. 2013. Parliaments and legislative activity. Wiesbaden: Springer VS.

Budge, Ian. 2015. Issue emphases, saliency theory and issue ownership: a historical and conceptual analysis. West European Politics 38(4):761-777. https://doi.org/10.1080/01402382.2015.1039374.

Burkhart, Simone, und Philip Manow. 2006. Kompromiss und Konflikt im parteipolitisierten Föderalismus der Bundesrepublik Deutschland. Zeitschrift für Politik 16(3):807-824.

Busemeyer, Marius R., Simon T. Franzmann, und Julian L. Garritzmann. 2013. Who owns education? Cleavage structures in the partisan competition over educational expansion. West European Politics 36(3):521-546. https://doi.org/10.1080/01402382.2012.753703.

Däubler, Thomas. 2008. Veto players and welfare state change: what delays social entitlement bills? Journal of Social Policy 37(04):683-706. https://doi.org/10.1017/S0047279408002274.

Debus, Marc, und Jochen Müller. 2013. Do voters' coalition preferences affect government formation? West European Politics 36(5):1007-1028. https://doi.org/10.1080/01402382.2013.796186.

Decker, Frank. 2011. Regieren im „Parteienbundesstaat“. Zur Architektur der deutschen Politik. Wiesbaden: VS.

Detterbeck, Klaus, und Wolfgang Renzsch. 2008. Symmetrien und Asymmetrien im bundesstaatlichen Parteienwettbewerb. In Parteien und Parteiensysteme in den deutschen Ländern, Hrsg. Uwe Jun, Melanie Haas, und Oskar Niedermayer, 39-55. Wiesbaden: VS.

Dewan, Torun, und Arthur Spirling. 2011. Strategic opposition and government cohesion in westminster democracies. American Political Science Review 105(2):337-358. https://doi.org/10.1017/ S0003055411000050.

Dolezal, Martin, Laurenz Ennser-Jedenastik, Wolfgang C. Müller, und Anna Katharina Winkler. 2014. How parties compete for votes: a test of saliency theory. European Journal of Political Research 53(1):57-76. https://doi.org/10.1111/1475-6765.12017.

Döring, Herbert. 1995. Parliaments and majority rule in Western Europe. Frankfurt: Campus.

Freitag, Markus, und Adrian Vatter (Hrsg.). 2008. Die Demokratien der deutschen Bundesländer: politische Institutionen im Vergleich. Opladen: Barbara Budrich.

Ganghof, Steffen. 2017. The empirical uses of theoretical models: the case of veto player theory. Political Studies Review 15(1):49-59. https://doi.org/10.1111/1478-9302.12098.

Ganghof, Steffen, Sebastian Eppner, Christian Stecker, Katja Heeß, und Stefan Schukraft. 2019. Do minority cabinets govern more flexibly and inclusively? Evidence from Germany. German Politics 28(4):541-561. https://doi.org/10.1080/09644008.2019.1635120.

Green-Pedersen, Christoffer. 2007. The growing importance of issue competition: the changing nature of party competition in western Europe. Political Studies 55(3):607-628. https://doi.org/10.1111/j. 1467-9248.2007.00686.x.

Green-Pedersen, Christoffer, und Peter B. Mortensen. 2014. Avoidance and engagement: issue competition in multiparty systems. Political Studies 63(4):747-764. https://doi.org/10.1111/1467-9248.12121.

Guinaudeau, Benjamin, und Isabelle Guinaudeau. 2020. (When) do elections set the legislative agenda? The extent and determinants of mandate responsiveness in Germany (1983-2016). Working Paper. 
Henkes, Christian, und Sascha Kneip. 2010. Von offener Neutralität zu (unintendiertem) Laizismus. Leviathan 38(4):589-616. https://doi.org/10.1007/s11578-010-0100-z.

Hesse, Konrad. 1962. Der unitarische Bundesstaat. Heidelberg: C. F. Müller.

Hildebrandt, Achim, und Frieder Wolf (Hrsg.). 2008. Die Politik der Bundesländer. Staatstätigkeit im Vergleich. Wiesbaden: VS.

Hörisch, Felix. 2018. Asylpolitik im Bundesländervergleich. Zeitschrift für Vergleichende Politikwissenschaft 12(4):783-803. https://doi.org/10.1007/s12286-018-0399-4.

Ismayr, Wolfgang. 2008a. Gesetzgebung im politischen System Deutschlands. In Gesetzgebung in Westeuropa. EU-Staaten und Europäische Union, Hrsg. Wolfgang Ismayr, 383-429. Wiesbaden: VS.

Ismayr, Wolfgang (Hrsg.). 2008b. Gesetzgebung in Westeuropa. EU-Staaten und Europäische Union, 1. Aufl., Wiesbaden: VS.

Jeffery, Charlie, und Arjan H. Schakel. 2012. Editorial: towards a regional political science. Regional Studies 47(3):299-302. https://doi.org/10.1080/00343404.2012.746443.

Jones, Bryan D., und Frank R. Baumgartner. 2005. The politics of attention: how government prioritizes problems. Chicago: University of Chicago Press.

Jun, Uwe. 1994. Koalitionsbildung in den deutschen Bundesländern: theoretische Betrachtungen, Dokumentation und Analyse der Koalitionsbildungen auf Länderebene seit 1949. Opladen: Leske + Budrich.

Kalke, Jens, und Peter Raschke. 2004. Regierungsmehrheit und Opposition in den bundesdeutschen Landtagen - eine quantitative Auswertung von Plenarprotokollen. In Kampf der Gewalten?, Hrsg. Everhard Holtmann, Werner J. Patzelt, 85-106. Wiesbaden: VS.

Graf Kielmansegg, Peter. 2013. Die Grammatik der Freiheit. Baden-Baden: Nomos.

König, Thomas, und Thomas Bräuninger. 2005. Gesetzgebung im Föderalismus. Speyer: Forschungsinstitut für Öffentliche Verwaltung.

Kriesi, Hanspeter, Edgar Grande, Martin Dolezal, Simon Bornschier, und Timotheos Frey. 2008. West European Politics in the Age of Globalization. Cambridge: Cambridge University Press.

Kropp, Sabine. 2001. Regieren in Koalitionen. Handlungsmuster und Entscheidungsbildung in deutschen Länderregierungen. Wiesbaden: Westdeutscher Verlag.

Kropp, Sabine. 2010. Kooperativer Föderalismus und Politikverflechtung. Wiesbaden: VS.

Laver, Michael, Kenneth Benoit, und John Garry. 2003. Extracting policy positions from political texts using words as data. American Political Science Review 97(2):311-331. https://doi.org/10.1017/ S0003055403000698.

Leber, Fabian. 2014. Landesgesetzgebung im neuen Bundesstaat. Handlungsmuster landespolitischer Akteure nach der Föderalismusreform 2006. Baden-Baden: Nomos.

Leunig, Sven. 2012. Die Regierungssysteme der deutschen Länder. Wiesbaden: Springer VS.

Leunig, Sven, und Hendrik Träger (Hrsg.). 2012. Parteipolitik und Landesinteressen. Der deutsche Bundesrat 1949-2009. Münster: LIT.

León, Sandra. 2014. How does decentralization affect electoral competition of state-wide parties? Evidence from Spain. Party Politics 20(3):391-402. https://doi.org/10.1177/1354068811436044.

Lijphart, Arend. 1999. Patterns of democracy. Government forms and performance in thirty-six countries. New Haven, London: Yale University Press.

Lipset, Martin Seymour, und Stein Rokkan. 1967. Cleavage structures, party systems and voter alignments. An introduction. New York: Free Press.

Manow, Philip, und Simone Burkhart. 2007. Legislative self-restraint under divided government in Germany, 1976-2002. Legislative Studies Quarterly 32(2):167-191.

Martin, Lanny W. 2004. The government agenda in parliamentary democracies. American Journal of Political Science 48(3):445-461. https://doi.org/10.1111/j.0092-5853.2004.00080.x.

Martin, Lanny W., und Georg Vanberg. 2004. Policing the Bargain: coalition government and parliamentary scrutiny. American Journal of Political Science 48(1):13-27. https://doi.org/10.1111/j.0092-5853. 2004.00053.x.

Martin, Lanny W., und Georg Vanberg. 2005. Coalition policymaking and legislative review. American Political Science Review 99(1):93-106. https://doi.org/10.1017/S0003055405051518.

Martin, Lanny W., und Georg Vanberg. 2011. Parliaments and Coalitions. The role of legislative institutions in multiparty governance. Oxford: Oxford University Press.

McGann, Anthony J. 2006. The logic of democracy. Reconciling equality, deliberation, and minority protection. Ann Arbor: University of Michigan Press.

Mielke, Siegfried, und Werner Reutter. 2012a. Länderparlamentarismus in Deutschland - Eine Bestandsaufnahme. In Länderparlamentarismus in Deutschland Geschichte - Struktur - Funktionen, Hrsg. Siegfried Mielke, Werner Reutter, 23-65. Wiesbaden: VS. 
Mielke, Siegried, und Werner Reutter (Hrsg.). 2012b. Länderparlamentarismus in Deutschland. Geschichte - Struktur - Funktionen. Wiesbaden: VS.

Miller, Bernhard. 2011. Der Koalitionsausschuss: Existenz, Einsatz und Effekte einer informellen Arena des Koalitionsmanagements. Baden-Baden: Nomos.

Müller, Jochen. 2013. On a short leash? Sub-national party positions between regional context and national party unity. Journal of Elections, Public Opinion \& Parties 23(2):177-199. https://doi.org/10.1080/ 17457289.2012.742092.

Müller, Wolfgang C., und Marcelo Jenny. 2004. „Business as usual“ mit getauschten Rollen oder Konflikt statt Konsensdemokratie? Parlamentarische Beziehungen unter der ÖVP-FPÖ-Koalition. Österreichische Zeitschrift für Politikwissenschaft 33:309-326.

von Oertzen, Jürgen. 2006. Das Expertenparlament. Abgeordnetenrollen in den Fachstrukturen bundesdeutscher Parlamente. Baden-Baden: Nomos.

Paasch, Jana, und Christian Stecker. 2021. When Europe hits the subnational authorities: the transposition of EU directives in Germany between 1990 and 2018. Journal of Public Policy, im Erscheinen. https:// doi.org/10.1017/S0143814X20000276.

Petrocik, John R. 1996. Issue ownership in presidential elections, with a 1980 case study. American Journal of Political Science 40(3):825-850. https://doi.org/10.2307/2111797.

Proksch, Sven-Oliver, und Jonathan B. Slapin. 2011. Parliamentary questions and oversight in the European Union. European Journal of Political Research 50(1):53-79. https://doi.org/10.1111/j.14756765.2010.01919.x.

Reus, Iris, und Stephan Vogel. 2018. Policy-Vielfalt zwischen den Bundesländern nach der Föderalismusreform I: Art, Ausmaß und Akteure. Zeitschrift für Vergleichende Politikwissenschaft 12(4):621-642. https://doi.org/10.1007/s12286-018-0406-9.

Reus, Iris, und Reimut Zohlnhöfer. 2015. Die christlich-liberale Koalition als Nutznießer der Föderalismusreform? In Politik im Schatten der Krise: Eine Bilanz der Regierung Merkel 2009-2013, Hrsg. Reimut Zohlnhöfer, Thomas Saalfeld, 245-272. Wiesbaden: Springer.

Reutter, Werner. 2007. Struktur und Dauer der Gesetzgebungsverfahren des Bundes. Zeitschrift für Parlamentsfragen 38(2):299-315.

Reutter, Werner. 2013. Zur Zukunft des Landesparlamentarismus. Der Landtag Nordrhein-Westfalen im Bundesländervergleich. Wiesbaden: Springer VS.

Robertson, David. 1976. Theory of party competition. London: John Wiley \& Sons Ltd.

Rohrschneider, Robert. 1994. Report from the laboratory: the influence of institutions on political elites' democratic values in Germany. American Political Science Review 88(4):927-941. https://doi.org/10. $2307 / 2082717$.

Roller, Edeltraud. 2004. Performanz. In Politische Theorie, Hrsg. Gerhard Göhler, Mattias Iser, und Ina Kerner, 297-314. Wiesbaden: VS.

Rudzio, Wolfgang. 2005. Informelles Regieren. Zum Koalitionsmanagement in deutschen und österreichischen Regierungen. Wiesbaden: VS.

Rudzio, Wolfgang. 2015. Das politische System der Bundesrepublik Deutschland. Wiesbaden: Springer VS.

Saalfeld, Thomas. 2000. Coalitions in Germany: stable parties, chancellor democracy and the Art of informal settlement. In Coalition governments in western Europe, Hrsg. Wolfgang C. Müller, Kaare Strøm, 32-85. Oxford: Oxford University Press.

Sack, Detlef. 2010. Europäisierungsdruck und Parteiendifferenz in den deutschen Bundesländern. Die Rechtsprechung des EuGH und die Novellierung von Tariftreueregelungen. Politische Vierteljahresschrift 51:619-642. https://doi.org/10.1007/s11615-010-0032-x.

Sack, Detlef, und Annette E. Töller. 2018. Einleitung: Policies in den deutschen Ländern. Zeitschrift für Vergleichende Politikwissenschaft 12(4):603-619. https://doi.org/10.1007/s12286-018-0408-7.

Sagarzazu, Iñaki, und Heike Klüver. 2017. Coalition governments and party competition: political communication strategies of coalition parties. Political Science Research and Methods 5(2):333-349. https:// doi.org/10.1017/psrm.2015.56.

Scharpf, Fritz W. 2006. Interaktionsformen. Akteurzentrierter Institutionalismus in der Politikforschung. Wiesbaden: VS.

Schneider, Gerald, Nadine Segadlo, und Miriam Leue. 2020. Forty-eight shades of Germany: positive and negative discrimination in federal asylum decision making. German Politics 29(4):564-581. https:// doi.org/10.1080/09644008.2019.1707810.

Schukraft, Stefan. 2011. Fraktionsgeschlossenheit auf Landesebene im Mehrebenen - Kontext - Der Einfluss des Föderalismus auf den Grad geschlossenen Abstimmungsverhaltens von Fraktionen in den deutschen Landesparlamenten. Politische Vierteljahresschrift 52(4):688-671. 
Schukraft, Stefan. 2019. Legilsativer Konflikt in den deutschen Ländern. Potsdam: Universitätsverlag Potsdam.

Schüttemeyer, Suzanne S. 1998. Fraktionen im deutschen Bundestag 1949-1997. Empirische Befunde und theoretische Folgerungen. Opladen: Leske + Budrich.

Schüttemeyer, Suzanne S. 2003. Vergleichende Parlamentarismusforschung. In Vergleichende Politikwissenschaft, Hrsg. Dirk Berg-Schlosser, Ferdinand Müller-Rommel, 201-221. Opladen: Leske + Budrich.

Sebaldt, Martin. 2009. Die Macht der Parlamente. Funktionen und Leistungsprofile nationaler Volksvertretungen in den alten Demokratien der Welt. Wiesbaden: VS.

Snyder, Richard. 2001. Scaling down: the subnational comparative method. Studies in Comparative International Development 36(1):93-110. https://doi.org/10.1007/BF02687586.

Spoon, Jae-Jae, Sara B. Hobolt, und Catherine E. de Vries. 2014. Going green: explaining issue competition on the environment. European Journal of Political Research 53(2):363-380. https://doi.org/10. 1111/1475-6765.12032.

Stecker, Christian. 2013. How effects on party unity vary across votes. Party Politics 22(5):791-802. https://doi.org/10.1177/1354068813509514.

Stecker, Christian. 2015. Parties on the chain of federalism. Position-taking and multi-level party competition in Germany. West European Politics 38(6):1305-1326. https://doi.org/10.1080/01402382.2015. 1029366.

Stecker, Christian. 2016. The effects of federalism reform on the legislative process in Germany. Regional \& Federal Studies 26(5):603-624. https://doi.org/10.1080/13597566.2016.1236334.

Strøm, Kaare, Wolfgang C. Müller, und Torbjörn Bergman. 2008. Cabinets and coalition bargaining: the democratic life cycle in Western Europe. Oxford, New York: Oxford University Press.

Switek, Niko. 2017. Grüne Farbenspiele. Neue Koalitionen von Bündnis 90/Die Grünen auf Länderebene. In Parteien unter Wettbewerbsdruck, Hrsg. Sebastian Bukow, Uwe Jun, 151-179. Wiesbaden: Springer.

Töller, Annette E., und Ulf Roßegger. 2018. Auswirkungen der Abweichungskompetenz der Länder. Zeitschrift für Vergleichende Politikwissenschaft 12(4):663-682. https://doi.org/10.1007/s12286-0180400-2.

Tsebelis, George. 1999. Veto players and law production in parliamentary democracies: an empirical analysis. American Political Science Review 93(3):591-608. https://doi.org/10.2307/2585576.

Tsebelis, George. 2002. Veto players. How political institutions work. Princeton: Princeton University Press.

Wachendorfer-Schmidt. 2005. Politikverflechtung im vereinigten Deutschland. Wiesbaden: VS.

Walgrave, Stefaan, Jonas Lefevere, und Michiel Nuytemans. 2009. Issue ownership stability and change: how political parties claim and maintain issues through media appearances. Political Communication 26(2):153-172. https://doi.org/10.1080/10584600902850718.

Wehling, Hans Georg. 2002. Die deutschen Länder. Geschichte, Politik, Wirtschaft. Opladen: Leske + Budrich.

Wenzelburger, Georg, Stefan Wurster, und Markus B. Siewert. 2020. Responsive Politikgestaltung in den deutschen Bundesländern? Versuch einer Systematisierung und Konzeption eines Forschungsprogramms. Zeitschrift für Vergleichende Politikwissenschaft 14(1):33-47. https://doi.org/10.1007/ s12286-020-00449-2. 